\title{
Modelling Outstanding Claims with Mixed Compound Processes in
}

\section{Insurance}

\author{
Gian Paolo Clemente ${ }^{1}$, Nino Savelli ${ }^{1}$, Diego Zappa ${ }^{2}$ \\ ${ }^{1}$ Dipartimento di Discipline Matematiche, Finanza Matematica ed Econometria, Università Cattolica del Sacro \\ Cuore, Largo Gemelli 1, 20123, Milano, Italy \\ ${ }^{2}$ Department of Statistical Sciences, Università Cattolica del Sacro Cuore, Largo Gemelli 1, 20123, Milano, Italy \\ Correspondence: Gian Paolo Clemente, Università Cattolica del Sacro Cuore, Largo Gemelli 1, 20123, Milano, \\ Italy.
}

Received: November 29, 2018

Accepted: January 14, 2019

Online Published: February 13, 2019

doi:10.5539/ibr.v12n3p123

URL: https://doi.org/10.5539/ibr.v12n3p123

\begin{abstract}
In general insurance, measuring the uncertainty of future loss payments and estimating the claims reserve are primary goals of actuaries. To deal with these tricky tasks, a broad literature is available on deterministic and stochastic approaches, most of which aims at straightforwardly modelling the overall claims reserve. In this paper by an extended, very general and reproducible case-study, we analyze the reserving process by attributing to each cell of the lower part of the run-off triangle a Compound mixed Poisson Process, calibrated upon both the numbers of claims and future average costs and considering as well the dependence among incremental claims. We provide analytically the moments of both incremental payments and the total reserve. Furthermore, we accordingly consider the probability distribution of the claims reserve, which is necessary for the assessment of the Risk Reserve capital requirement in a Solvency II framework. To test the impact of the model under different scenarios, insurers and lines of business, the case study is thoroughly analyzed by exploiting the Fisher-Lange average cost method.
\end{abstract}

Keywords: stochastic models for claims reserve, reserve risk, collective risk model, average cost methods, Solvency II

\section{Introduction}

The estimation of liabilities for outstanding claims is a key issue in general insurance. For a long time deterministic methods, e.g. the worldwide used Chain-Ladder (CL) algorithm (see for instance Friedland (2010)), have been applied to obtain the value of these provisions. Under the Solvency II framework (see European Commission $(2009,2013,2015))$ and the new international accounting principles in insurance (e.g. IAS and IFRS), the evaluation of the uncertainty of future claims obligations is also required. To achieve this aim, stochastic models have been introduced. Our contribution belongs to these approaches. To understand the innovations we are proposing a short survey of this topic is necessary.

The literature on claim reserving is very wide. Though limited, we start this survey by commenting on the research by Thomas Mack, one of the pioneers in this research field. He proposed in (Mack, 1993) a non-parametric way of computing the standard error of the CL method by separating uncertainty on reserve estimation due to the data (Process Error) from uncertainty deriving from the method used to estimate parameters (Estimation Error). Later, in a parametric context, Generalized Linear Models (GLM) have been used to provide both appropriate variability of the reserve estimate and a general inferential set up based on the maximum likelihood approach (see Renshaw and Verrall (1998)). GLM residuals have led the way to a semiparametric bootstrapping procedure. By fitting appropriately incremental payments, this methodology (see England (2002); England and Verrall (1999)) re-samples from the corresponding adjusted Pearson residuals. Practitioners often use a GLM Over-Dispersed Poisson (ODP) model (see England and Verrall (2002)), since this provides estimates $\operatorname{close}^{i}$ to the standard Chain-Ladder approach used worldwide. The probability distribution of outstanding claims can be easily derived from the Monte-Carlo procedure. Recent developments are presented in Pinheiro et al. (2003) and Tee et al. (2017) ${ }^{\mathrm{ii}}$. Further generalization of GLMs in loss reserving exploits the richness of the Tweedie family of distributions (see Peters et al. (2009); Wüthrich (2003)). For extensions to a flexible multivariate approach to consider the dependence between claims of different lines of business (LoBs) 
see Avanzi et al. (2016). Other deterministic methods, such as Bornhuetter-Ferguson, have been recently integrated with a stochastic structure based on a Bayesian approach (England and Verrall, 2006; England et al., 2012), aiming at measuring the prediction error of the reserve estimate (Alai et al., 2009; Saluz et al., 2011).

Seeing that so many methods have been developed, the International Actuarial Association (2004) suggested a standard methodology based on a paper by Meyers et al. (2003) focusing on the collective approach typically used for premium risk. This methodology assumes that the overall reserve of a single LoB follows a compound mixed Poisson process. The assumptions are that open claim counts are Negative Binomial distributed and that the severity of outstanding claims has a known mean and coefficient of variation, whatever the type accident and/or the development year (see also Martìnez-Miranda et al. (2015) and Meyers (2008) for similar approaches). The drawback to this approach is that it directly estimates the overall claims reserve without decomposing it into accident years. This seems too naïve since empirical studies have shown that significant differences exist among claims of different accident and development years. Furthermore, actuarial practice suggests considering the time dependency of average payments (see, e.g., Friedland (2010) for more details).

Our research falls within this framework. The point is how to model the claims of different accident and development years by considering separately consistent estimates of the average cost and the claim counts. To achieve this aim, we extend the IAA approach by modelling each single cell of the lower run-off triangle through a Collective Risk Model (CRM) based on a Compound mixed Poisson Process, where both the number of claims and the severities are mixed random variables. So doing, from an actuarial point of view we add further flexibility to the model. By adding parameter uncertainty for numbers and claims, we consider as well the correlation between incremental claims within the triangle. This point is not covered by standard ODP methodologies (see below for details). In this paper by an extended, very general and reproducible case-study, we show the effect of this parameterization. We obtain the analytical formulae of the moments of the reserve distribution by assuming only the knowledge of the main moments of the two random variables (the number and the claim size of future payments) involved in the CRM. Similar to other papers on this topic, Monte Carlo simulations are used to derive the outstanding claims distributions for each accident year, for the overall reserve, and for the next calendar year. This technique is almost compulsory since the number of claims involved in the convolution Poisson process is so large that the exact distribution is numerically unfeasible. Furthermore, since a one-year time horizon for capital requirement evaluation is prescribed by the Solvency II project (European Commission, 2009), we compute the one-year reserve risk by adapting the re-reserving method, assuming CRM as the underlying stochastic methodology (see Diers (2009) and Ohlsson and Lauzeningks (2008) for "one-year" approaches based on the Bootstrap-CL method). In this way, we are also able to simulate the claims development result and thus evaluate the capital requirement for the reserve risk.

To test the impact of our proposal with respect to other methods, we have applied it to different insurers and to several LoBs. We explore the effects on the claims reserve (such as the Best Estimate plus Risk Margin according to the Solvency II framework) and on the capital requirement obtained by either the Internal Model or the Standard Formula (both Market-Wide and Undertaking Specific approaches). We calibrate the model's parameters using real data based on the number of claims to be paid and the future average costs for each cell of the triangle that is to be estimated. To achieve this aim, we will make use of the Fisher-Lange method (see Fisher and Lange (1973) and Ottaviani (1983) for a slightly modified version). At present, this method is available in the literature only in a deterministic version. We have extended it to a stochastic framework as well.

The paper is organized as follows. Section 2 presents the general CRM structure of our proposal and the main analytical results. Section 3 focuses on parameter calibration and the Fisher-Lange method used to estimate the mean of the random variables involved in the procedure. In Section 4, the model has been extended with the aim of evaluating the capital requirement in a framework consistent with the Solvency II approach. In Section 5, a detailed case study based on real data from the Motor Third-Party Liabilities line of business is reported. Claims reserve distribution is compared to the popular ODP bootstrap method. We provide comparative scenarios related to other lines of business. Finally, we assess the aggregate capital requirement both by assuming linear correlation and by exploiting different copula functions. Conclusions follow.

\section{Methodology}

In insurance, claims are typically classified according to when they are incurred, called the accident year (AY), and when they are paid, called the development year (DY). CRM, typically used to evaluate premium risk, exploits collective risk theory in order to estimate incremental claims, i.e. the sum of all the claims incurred in a specific AY but not yet paid in a specific DY. It models aggregate claims by a random sum of random claims, each identically distributed according to a claim-size distribution. In this context, some authors have shown how 
to obtain the convolution under some limited assumptions (see Daykin et al. (1993)) and how to find an approximate distribution of the total reserve distribution (Daykin et al., 1993; Klugman et al., 2008). We extend those results by finding the moment generating function (m.g.f.) of the incremental claims when both the number of claims and the losses are mixed random variables ${ }^{\text {iii }}$ (r.v.), showing the effect on claim reserve of correlation among incremental claims.

The overall amount of claims and the corresponding number of claims for each AY and DY are typically reported in matrices of dimension $t \times t^{+}$, where rows $(i=1, \ldots, t)$ represent AY and columns $\left(j=1, \ldots, t^{+}\right)$are DY. In the actuarial field, these matrices are called Run-Off Triangles. They report claims incurred in the i-th AY and paid in the $\mathrm{j}$-th DY (namely, in the calendar year $i+j-1$ ). Therefore, the data are available only in the upper left part of the Run-Off matrix (i.e. only for $1 \leq j \leq t^{+}$and $i+j \leq t+1$ ) and will be denoted by $X_{i, j}$.

The main interest of actuaries is the estimation of future payments, i.e. the claims that must be allocated to each cell of the matrix where data are still missing. For $1 \leq j \leq t^{+}$and $i+j>t+1$, these cells include unknown values from appropriate $\tilde{X}_{i, j}$ r.v.s.

Note that frequently columns are not equal to rows because of payments incurred at time $i=1$ that still have to be paid. Hence, we have a tail in the triangle (i.e. $t^{+}=t+1$ ). Instead, we have $t^{+}=t$ when all claims from the first AY are fully run-off by the end of the last observed DY $(j=t)$. In this case, the payments in the triangle represent the complete run-off of claims. In order to build up a stochastic model based on the Compound Poisson Process, we assume that, in each cell of the lower part, the incremental paid claim cost is equal to the aggregate claim amount:

$$
\tilde{X}_{i, j}=\sum_{h=1}^{\widetilde{N}_{i, j}} \tilde{Z}_{i, j, h}
$$

where:

- $\quad \widetilde{N}_{i, j}$ is typically assumed to be Poisson distributed with parameter $n_{i, j}$. In this paper, we consider a mixed Poisson Process. To include the uncertainty due to parameter estimation, market heterogeneity and external background factors (such as weather, economic conditions, etc.), $n_{i, j}$ is multiplied by a structure r.v. $\tilde{q}_{i, j}$ (see Daykin et al. (1993) for an application of structure variables in premium risk modelling). In the next section, we will assume $\tilde{q}_{i, j}$ to be a Gamma r.v. with mean equal to 1 (i.e. the shape and the scale parameters are equal, for example, to $q$ ) and standard deviation equal to $\sigma_{\tilde{q}_{i, j}}$. The constraint $E\left(\tilde{q}_{i, j}\right)=1$ assures that the average number of claims stays at the expected level. Hence, $\tilde{q}_{i, j}$ will impact the variability of $\widetilde{N}_{i, j}$. In general, if we refer to the missing lower part of the triangle, $n_{i, j}$ represents the expected number of claims in the cell $(i, j)$. The average paid claim is given by averaging costs $m_{i, j}=E\left(\tilde{X}_{i, j} / \widetilde{N}_{i, j}\right)$.

- $\quad \tilde{Z}_{i, j, h}$ is the random amount (loss) of the $h$-th claim settled after $j-1$ years and occurred in accident year $i$. As with the claim count variable, we measure the parameter uncertainty of a single claim cost by means of a structure variable having an impact only on variability. This uncertainty is described by a multiplicative random variable $\tilde{r}_{i, j}$, assumed to be Gamma distributed with a mean equal to 1 (therefore, the parameters are both equal, for example, to $r$ for the same reason reported above for $\widetilde{N}_{i, j}$ ) and a standard deviation of $\sigma_{\tilde{r}_{j}}$. In each cell $(i, j)$ in the lower part of the triangle the claim sizes $\tilde{Z}_{i, j, h}$ for $h=1,2, \ldots$, are assumed non-negative i.i.d. random variables. Furthermore, as customary in actuarial applications (see Daykin et al. (1993)), the mutual independence of the claim count and claim costs within a single cell is also assumed. Also structural variables $\tilde{q}_{i, j}$ and $\tilde{r}_{i, j}$ are assumed independent.

Within this framework, we can obtain the exact moments and cumulants of $\tilde{X}_{i, j}$ for any $i, j$ (see Savelli and Clemente (2010) for some preliminary results). Given a generic $\tilde{Y}$, let $M_{\tilde{Y}}(t)$ and $F_{\tilde{Y}}(y)$ be the m.g.f. and the cumulative distribution function, respectively. To keep notation as simple as possible, for the next few steps we drop subscripts and use $\tilde{X}=\tilde{X}_{i, j}$. Next, let $M_{\tilde{X}}(t)$ be the m.g.f. referred to the cell $i, j$. We have: 


$$
M_{\tilde{X}}(t)=E\left[e^{t \tilde{X}}\right]=\sum_{k=0}^{\infty} E\left[e^{t\left(\tilde{Z}_{1}+\cdots+\tilde{Z}_{k}\right)} \mid \widetilde{N}=k\right] \operatorname{Pr}[\widetilde{N}=k] .
$$

Recalling that by assumption $\tilde{Z}_{h}$ are i.i.d., then

$$
M_{\tilde{X}}(t)=E_{\widetilde{N}}\left[E_{\tilde{Z}}\left[e^{t \tilde{Z}}\right]^{k} \mid \widetilde{N}=k\right]=E_{\widetilde{N}}\left[M_{\tilde{Z}}[t]^{k} \mid \widetilde{N}=k\right]=M_{\widetilde{N}}\left[\ln M_{\tilde{Z}}[t]\right] .
$$

If $\widetilde{N}$ is a mixed Poisson r.v. as described above (see (1)), then:

and

$$
M_{\widetilde{N}}(t)=E\left[e^{\widetilde{N} t}\right]=E_{\tilde{q}}\left[M_{\widetilde{N}}(t \mid \widetilde{q}=q)\right]=E\left[e^{\tilde{q} n\left(e^{t}-1\right)}\right]=M_{\tilde{q}}\left(n\left(e^{t}-1\right)\right)
$$

$$
M_{\tilde{X}}(t)=M_{\tilde{q}}\left(n\left(M_{\tilde{Z}}[t]-1\right)\right) .
$$

If, for example ${ }^{\text {iv }}, \tilde{Z} \sim G a(\alpha, \beta)$, and we assume to multiply the shape parameter $\alpha$ by $\tilde{r}$ distributed as a Gamma r.v. with $E(\tilde{r})=1$ and standard deviation $\sigma_{\tilde{r}}$, we have the mixed r.v. $\tilde{Z}_{\tilde{r}} \sim G a(\alpha \tilde{r}, \beta)$.

Recalling that $M_{\tilde{Z}}(t)=\left(\frac{\beta}{\beta-t}\right)^{\alpha}$ with $\beta>t$, we have:

$$
M_{\tilde{z}_{\tilde{r}}}(t)=E\left[e^{\tilde{z}_{\tilde{r}} t}\right]=E_{\tilde{r}}\left[M_{\tilde{Z}}(t \mid \tilde{r}=r)\right]=\int_{0}^{\infty} e^{r \alpha \ln \left(\frac{\beta}{\beta-t}\right)} d F_{\tilde{r}}(r)=M_{\tilde{r}}\left(\alpha \ln \left(\frac{\beta}{\beta-t}\right)\right) .
$$

Combining (2) and (3), we obtain:

$$
M_{\tilde{X}}(t)=M_{\tilde{q}}\left(n\left[M_{\tilde{r}}\left(\alpha \ln \left(\frac{\beta}{\beta-t}\right)\right)-1\right]\right)=q^{q}\left(q-n r^{r}\left(r-\alpha \ln \left(\frac{\beta}{\beta-t}\right)\right)^{-r}+n\right)^{-q} .
$$

Taking the derivative in $\mathrm{t}=0$ :

$$
\begin{gathered}
\left.M_{\tilde{X}}^{\prime}(t)\right|_{t=0}=n E(\tilde{Z})=n m=E(\tilde{X}) \\
\left.M_{\tilde{X}}^{\prime \prime}(t)\right|_{t=0}=n \frac{\alpha}{\beta^{2}}+n\left(r^{-1}+1\right) m^{2}+n^{2} m^{2}\left(q^{-1}+1\right) \\
=n \sigma_{\tilde{Z}}^{2}+n m^{2} \sigma_{\tilde{r}}^{2}+n m^{2}+n^{2} m^{2} \sigma_{\tilde{q}}^{2}+n^{2} m^{2}
\end{gathered}
$$

which gives:

$$
\sigma_{\tilde{X}}^{2}=n a_{2, \tilde{Z}}+n m^{2} \sigma_{\tilde{r}}^{2}+n^{2} m^{2} \sigma_{\tilde{q}}^{2}
$$

where $a_{2, \tilde{z}}$ is the second raw moment of $\tilde{Z}$.

We deduce that the standard deviation is affected by three components: the first refers to the process error related to claim count and claim size, while the others describe the parameter uncertainty of the claim size and the claim count, respectively. The coefficient of variation of $\tilde{X}_{i, j}$ is:

$$
C V\left(\tilde{X}_{i, j}\right)=\sqrt{\frac{a_{2, \tilde{z}_{i, j}}}{n_{i, j} m_{i, j}^{2}}+\frac{\sigma_{\tilde{r}_{j}}^{2}}{n_{i, j}}+\sigma_{\tilde{q}_{i, j}}^{2}}=\sqrt{\frac{1+c_{\tilde{z}_{j}}^{2}+\sigma_{\tilde{r}_{j}}^{2}}{n_{i, j}}+\sigma_{\tilde{q}_{i, j}}^{2}}
$$

As $n_{i, j}$ approaches infinity, the coefficient of variation is affected only by the standard deviation of the structure variable $\tilde{q}_{i, j}$, while the standard deviation of the random variable $\tilde{r}_{j}$ has a decreasing effect on variability as $n_{i, j}$ increases. The comparative scenarios reported in Savelli and Clemente (2010) confirm that in most cases the parameter uncertainty on claim size contributes in a limited way to the overall variability with respect to $\tilde{q}_{i, j}$.

Now, we extend these results to derive the cumulants of claims reserve, considering also the correlation between the different cells in the lower triangle containing the number of claims and the average claims cost of the same DY. A realistic assumption regarding the number of claims is $\tilde{q}_{i, j}=\tilde{q} \forall i, j$ and it will be assumed that $\tilde{r}_{i, j}=\tilde{r}_{j} \forall i$. We use a specific uncertainty for each DY, since the claims costs for each $j$ are estimated separately.

The main characteristics of the claims reserve, $\tilde{R}$, can be derived. As expected, the average claims reserve is straightforwardly equal to: 


$$
E(\tilde{R})=\sum_{i=1}^{t} E\left(\tilde{R}_{i}\right)=\sum_{i=1}^{t} \sum_{j=t-i+2}^{t^{+}} E\left(\tilde{X}_{i, j}\right)=\sum_{i=1}^{t} \sum_{j=t-i+2}^{t^{+}} n_{i, j} m_{i, j} .
$$

We can explicitly compute the covariance between each pair of incremental payments as:

$$
\begin{gathered}
\operatorname{Cov}\left(\tilde{X}_{i, j}, \tilde{X}_{h, k}\right)=E\left[E\left(\tilde{X}_{i, j} \tilde{X}_{h, k} \mid \tilde{q}=q, \tilde{r}_{i, j}=\tilde{r}_{j}, \tilde{r}_{h, k}=\tilde{r}_{k}\right)\right]-E\left(\tilde{X}_{i, j}\right) E\left(\tilde{X}_{h, k}\right)= \\
=\left\{\begin{array}{cc}
\left(E\left(\tilde{q}^{2}\right)-1\right)\left(n_{i, j} m_{i, j} n_{h, k} m_{h, k}\right)=\sigma_{\tilde{q}}^{2} n_{i, j} m_{i, j} n_{h, k} m_{h, k} & \text { for } j \neq k \\
\left(E\left(\tilde{q}^{2}\right) E\left(\tilde{r}_{j}^{2}\right)-1\right) n_{i, j} m_{i, j} n_{h, j} m_{h, j} & \text { for } j=k
\end{array}\right.
\end{gathered}
$$

In particular, for $j=k$ (i.e. between AYs) the correlation coefficient is:

where $\sigma_{\tilde{X}_{i, j}}^{2}=\left(n_{i, j} a_{2, \tilde{z}_{i, j}}+n_{i, j} m_{i, j}^{2} \sigma_{\tilde{r}_{j}}^{2}+n_{i, j}^{2} m_{i, j}^{2} \sigma_{\tilde{q}}^{2}\right)$.

$$
\rho\left(\tilde{X}_{i, j}, \tilde{X}_{h, k}\right)=\frac{\left(E\left(\tilde{q}^{2}\right) E\left(\tilde{r}_{j}^{2}\right)-1\right) n_{i, j} m_{i, j} n_{h, j} m_{h, j}}{\sigma_{\tilde{X}_{i, j}} \sigma_{\tilde{X}_{h . k}}}
$$

Since it was assumed that $E(\tilde{q})=E\left(\tilde{r}_{j}\right)=1$, and since $\operatorname{Var}(\tilde{q})=E\left(\tilde{q}^{2}\right)-1 \geq 0$ (the same holds for $\operatorname{Var}\left(\tilde{r}_{j}\right)$ ), it follows that $E\left(\tilde{q}^{2}\right) E\left(\tilde{r}_{j}^{2}\right) \geq 1$ and, therefore, $\rho\left(\tilde{X}_{i, j}, \tilde{X}_{h, k}\right) \geq 0$. This term is not considered by the IAA approach; it contributes to the reserving process by inflating the overall variance, that is:

$$
\begin{aligned}
\sigma^{2}(\tilde{R})=\sum_{i=1}^{t} \sum_{j=t-i+2}^{t^{+}} \sigma^{2}\left(\tilde{X}_{i, j}\right)+\sum_{i, j} \sum_{\substack{h, k \\
h \neq i \vee j \neq k}} \operatorname{Cov}\left(\tilde{X}_{i, j}, \tilde{X}_{h, k}\right) \\
=\sum_{i=1}^{t} \sum_{j=t-i+2}^{t^{+}}\left(n_{i, j} a_{2, \tilde{z}_{i, j}}+n_{i, j} m_{i, j}^{2} \sigma_{\tilde{r}_{j}}^{2}+n_{i, j}^{2} m_{i, j}^{2} \sigma_{\tilde{q}}^{2}\right) \\
+\sum_{i, j} \sum_{\substack{h, k \\
j \neq k}} n_{i, j} m_{i, j} n_{h, k} m_{h, k} \sigma_{\tilde{q}}^{2}+\sum_{i, j} \sum_{\substack{h, k \\
h \neq i \vee j=k}}\left(E\left(\tilde{q}^{2}\right) E\left(\tilde{r}_{j}^{2}\right)-1\right) n_{i, j} m_{i, j} n_{h, k} m_{h, k}
\end{aligned}
$$

For each DY and AY, the first term sums the variances of the single cells in the lower triangle. The second term considers the dependency between claims counts for different DY. The third addendum introduces an implicit correlation between AYs due to the structure variables that jointly affect the whole triangle. It is clear that the variance is not lower than the sum of the variances of the claim payments in each cell:

$$
\sigma^{2}(\tilde{R}) \geq \sum_{i=1}^{t} \sum_{j=t-i+2}^{t^{+}} \sigma^{2}\left(\tilde{X}_{i, j}\right) .
$$

The results can be used in closed form to find the distribution of outstanding claims following methodologies based on approximation: for example, Normal Approximations, the recursive Panjer method, the fast Fourier transform, etc. (Daykin et al., 1993; Klugman et al., 2008), or using simulation methods. The latter approach will be used later to estimate the distribution, since it is much more numerically robust and computationally feasible. Moreover, this approach is almost compulsory when single claims for each cell of the run-off triangle are available. In this case, we may customize each time event with its proper distribution function by an appropriate fitting procedure. This extends the capability of standard CRM by adding a mixture of distributions (not necessarily of the same family) and by solving the not-rare cases where some accident, development or calendar years are affected by specific events (e.g. large losses, a change in the claims inflation rate, a change in legislation, etc.) or commercial strategies.

\section{Parameter Estimates Using the Fisher-Lange Method}

As mentioned above, to apply a CRM we need both the expected number of the paid claims triangle and, correspondingly, its expected cost. To estimate the claims reserve we have chosen the Fisher-Lange Method (F-L) (Fisher and Lange, 1973; Ottaviani, 1983) since this allows for a separate description of the number and the cost of claims and it is not based only on the aggregate payments. Alternatives are available in the literature, such as Paid or Incurred Chain-Ladder, Bornhuetter-Ferguson. All of these are widely used in actuarial practice; however, 
they do not possess the desirable property of F-L.

F-L belongs to frequency-severity methods (see Friedland (2010)) where ultimate claims are forecasted by multiplying the estimate of the ultimate number of claims by the estimate of the ultimate average value. The following is a sketch of the F-L method. The incremental number of claims $\left(n_{i, j}\right)$ is supposed to be related to the number of reserved claims at the valuation date $\left(n_{r_{i, t-i+1}}\right)$ through the coefficients aliq $_{j}$ and $v_{i, j}$ as follows:

$$
n_{i, j}=n_{r_{i, t-i+1}} \cdot \operatorname{aliq}_{j} \cdot v_{i, j}
$$

where:

- $\operatorname{aliq}_{j}$ is the percentage of claims reserved having a payment in future years. At the valuation date, it is the portion of claims at reserve having effectively a payment in the future, regarding implicitly either nil, reopened and IBNR claims. Through these percentages (one for each duration), estimated on the basis of past data and applied to $n_{r_{i, t-i+1}}$, we obtain the estimate of the number of claims to be paid in the future for each accident year $i$.

- $v_{i, j}$ is the claim settlement speed, representing the timing of the claims count payments estimated to have effectively a payment in the future. In practice, we need an estimate concerning the timing of the paid claims count in the future for each separate accident year. This can be obtained by the number of claims effectively paid in the last calendar year for different development years $\left(j=2, \ldots, t^{+}\right)$, but where the origin of different accident years has to be made homogeneous (by a proportionality rule based on the number of reported claims). Moreover, the average of the last three calendar years is often taken into account to assure a greater stability of the estimate (this option will be adopted in our numerical examples).

To estimate expected average paid costs $\left(m_{i, j}\right)$ for each cell of the future triangle, including future inflation, four steps are needed:

1. adjust the historic payments (set in the upper part of the run-off triangle) to the current value of the last calendar year available by annual effective economic inflation rates (i.e. endogenous inflation is not taken into account);

2. compute the average paid claims for each cell of the triangle obtained at step 1;

3. select for each development year the average cost $\left(\bar{m}_{j}\right)$ as a simple average (or alternatively as a weighted average, having the number of effective paid claims as weights) of all/part of available average costs for the same duration (average by column) ${ }^{v}$. The simple average will be adopted in our numerical examples;

4. inflate the chosen average cost of each column, according to the total claim inflation (either endogenous and exogenous), $i_{h}$, expected in the future:

$$
m_{i, j}=\left\{\begin{array}{l}
\bar{m}_{j} \cdot \prod_{h=t+2}^{(i+j)}\left(1+i_{h}\right) \text { if } j<t^{+} \\
\bar{m}_{j} \cdot \prod_{h=t+2}^{(i+t+1)}\left(1+i_{h}\right) \text { if } j=t^{+}
\end{array} .\right.
$$

In this way we obtain both the expected number of paid claims $\left(n_{i, j}\right)$ and average paid cost $\left(m_{i, j}\right)$ for each cell of the future triangle.

Calibrating the parameters according to F-L, the average of the CRM model equals the F-L Best Estimate. It is noteworthy that, by using results for $\sigma(\tilde{R})$, we also obtain a measure of variability of the F-L estimate (or of any other deterministic method based on the number and the cost of claims), which otherwise would not have been available.

\section{Main Impact in a Solvency II Framework}

In actuarial science, any new method proposed for the distribution of claims reserve until the complete run-off of claims must be accomplished through comments on its impact on the uncertainty of the valuation of this liability. To address this issue, we must consider that insurers deal with the Solvency II framework in order to assess the capital requirement for reserve risk. In this case, models must be adapted in order to catch the "one-year" risk, as 
detailed in the next Section. This view assures consistency with the one-year time horizon prescribed by Solvency II for capital assessment purposes. The Solvency II Directive (see European Commission $(2009,2015)$ ) codifies and harmonises European Union (EU) insurance legislation. It primarily concerns the amount of capital that an insurer in the EU must hold to reduce the risk of insolvency. Both quantitative and qualitative measures for the calculation of technical provisions and capital requirements are set out; these came into force at the beginning of 2016. Focusing on reserve risk, either a Standard Formula (SF) or an Internal Model (IM) is allowed for capital estimation. The IM capital requirement in $t=0$ at the $\alpha$ confidence level $\left(S C R^{\alpha}\right)$ is obtained as the difference between the quantile of the distribution of the insurer obligations at year end of the next accounting year $\left(R_{1}^{-}\right)$, opportunely discounted at time zero with the risk-free discount factor $v_{1}$, and the best estimate ${ }^{\mathrm{vi}}$ at time $0\left(R_{0}\right)$ :

$$
S C R^{\alpha}=v_{1} \operatorname{VaR}_{\alpha}\left(\tilde{R}_{1}^{-}\right)-R_{0}
$$

where $v_{1}$ is the one-year risk-free discount factor provided by EIOPA. $\tilde{R}_{1}^{-}$, i.e. the r.v. of the insurer's obligation at the end of the following year, is the convolution of the payments that occurred in year 1 (i.e. the elements located on the next diagonal of the triangle) and the best estimate at time $t=1$, given the additional information available during the year.

The tricky task is the estimation of $\operatorname{VaR}_{\alpha}\left(\tilde{R}_{1}^{-}\right)$. At present, all the methodologies provided in the literature are applied to stochastic models based on the C-L method. In particular, the "re-reserving" method (Diers, 2009; Ohlsson and Lauzeningks, 2008) derives the distribution of $\tilde{R}_{1}^{-}$when the ODP-Bootstrap is used. We can adapt this fruitful method to our context.

1. The first step is to assess the opening reserve estimate $R_{0}$, which is calculated with the underlying reserving model, in our case the F-L described in Section 3 and the C-L method when Bootstrap is applied.

2. Next, it is necessary to simulate the number of claims and incremental payments for the next calendar year $t=1$, for each former accident year, using the underlying ultimate stochastic reserving process (i.e. the CRM model in our case). By so doing, we only have to simulate the subsequent diagonal in the development triangles. The sum of the claim payments on the simulated diagonal corresponds to the following year's payments.

3. The results in Step 2 are used to carry out a best estimate for the closing reserve in each simulation path, according to the underlying reserving model. This process is called re-reserving.

$\tilde{R}_{1}^{-}$is the convolution of the distributions derived from the second and the third step. As a result, we can compute both the quantile of the discounted one-year technical result and the SCR.

\section{Case Study}

The case study refers to claims on the Motor Third Party Liability (MTPL) line of business of two Italian insurance companies. The dataset covers 12 consecutive accounting years ${ }^{\text {vii }}$. For confidentiality, the data has been modified applying proportionality coefficients and the fictitious company names SIFA and AMASES have been used. SIFA is a small-medium-sized company, whereas AMASES is a company roughly 10 times larger (in terms of claims reserve volume).

Discounted Best Estimate obtained by the classical Chain-Ladder method is equal to roughly $€ 233$ (million) for SIFA and $€ 2.566$ (billion) for AMASES. The datasets report the number of paid and reserved claims, the number of closed and reopened claims, the number of reported claims, and the incremental payments and booked reserves amounts. Run Off triangles for MTPL and other LoBs are available on the website of the corresponding Author. As usual in an MTPL reserving context, data are summed up in 12x12 triangles with a tail. For the first AY, a complete run-off period longer than DY 12 is present. A tail must subsequently be added. In these triangles, the tails are the level of statutory reserve fixed by the companies. As is customary in the actuarial literature ${ }^{\text {viii }}$, the tail (12+) will be added to the payments of the last development year (12), reconciling the triangle to a standard regular shape.

We have initially applied our proposal as explained in Section 2. Specifically, for each missing cell of the Run Off triangle the number of claims is supposed to be distributed as a Negative Binomial, achieved by mixing a Poisson with a Gamma random variable. The average of the Poisson was set to $n_{i, j} \cdot \tilde{q}$, where $n_{i, j}$ is estimated by the F-L algorithm (see Section 3) and $\tilde{q}$ is supposed to be Gamma distributed with $E(\tilde{q})=1$ and $\sigma_{\tilde{q}}$ equals to 0.03 and 0.08 for AMASES and SIFA, respectively. Standard deviations have been estimated by taking into account the implicit correlation between claim counts derived by the Mack prediction error formula (see Mack (1993)).

Furthermore, by fitting the empirical distribution of the severities, we picked up the Gamma distribution to describe the cost of claims ${ }^{\text {ix }}$. To guarantee coherence with the expected overall cost computed with the other 
method, the mean was set equal to the average cost of the cell, i.e. $m_{i, j}$ (estimated by F-L as described in Section 3). The standard deviation is computed by knowing the coefficients of variation $c_{\tilde{z}_{j}}$ of the observed claims for each DY; the standard deviation depends on both the LoB of interest and the company's portfolio characteristic. An accurate estimate of $c_{\tilde{Z}_{j}}$ is a key issue, since the standard deviation depends on it. This estimation is not an obvious task. In general, data from the Claim DataBase of the company and for each development year is necessary. This data is often only available to internal actuaries and is rather difficult to obtain, even for an external actuary (e.g. involved in a take-over evaluation). To consider parameter uncertainty as described in Section 2, we have assumed that $c_{\tilde{Z}_{j}}$ is affected by a structure variable $\tilde{r}_{j}$ distributed according to a Gamma with $E(\tilde{r})=1, \sigma_{\tilde{r}}=0.03$ for both companies, derived by applying the Mack prediction error formula to claim costs (see Mack (1993)). As our perspective is based on uncertainty measurement, our comments will mainly focus on the impact of the method on the variability of the reserves. First, let us consider some differences between the claims reserve for each accident year. The coefficient of variation and the skewness by AY, from 1 to 12, are reported in Figure 1. We focus here on the second and third-order moments since the best estimate equals the F-L deterministic method claims reserve estimate. Differences between companies are very noticeable in earlier accident years. Both moments fall with a faster rate for SIFA, the smallest insurer.
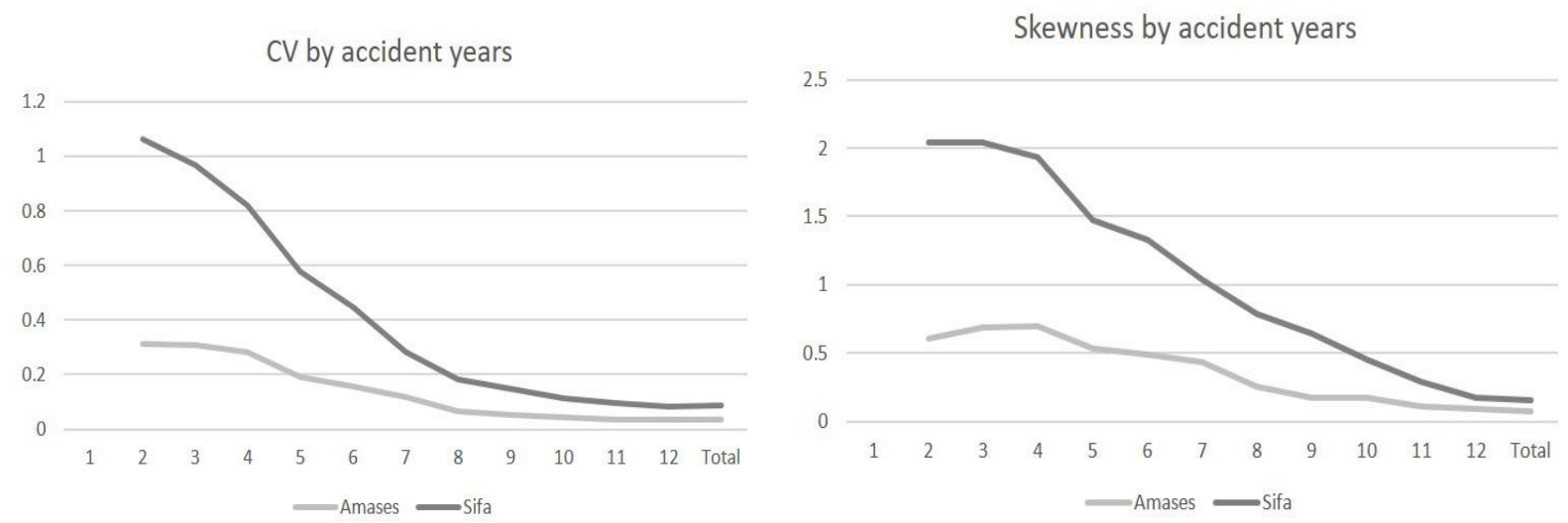

Figure 1. Coefficient of variation and Skewness of claims reserve distributions for accident year (CRM model)

For SIFA the overall (total) coefficient of variation equals 0.087, and there is not-negligible skewness $(+0.15)$. There is a lower variability and skewness, 0.033 and +0.06 , respectively, for AMASES. The analysis of the asymmetry of probability distribution of the claims reserve is a big issue in the actuarial field, since both non-proportional reinsurance strategies and capital requirements (see results below) are strictly dependent on the skewness of the distribution. In general, the shape of this distribution also indicates how much an insurer is exposed towards large or extreme losses. It is noteworthy that the CV and skewness for the overall claims reserve are low with respect to the single AY. This is due to the significant contribution (roughly $30 \%$ of total reserve) of the current generation (i.e. 12), where estimates are very low.

In order to propose some sensitivities, we compared the CRM approach to the Bootstrapping method (see England and Verrall (1999)) widely used by practitioners. The last approach assumes the application of the Chain-Ladder or ODP methods and the corresponding adjusted Pearson residuals with a constant scale parameter for different development years along with the assumption of incremental payments generated by a given distribution, e.g. Gamma, LogNormal.

Table 1 compares the coefficient of variation and the skewness given by the ODP Bootstrap and CRM methods. In general, CRM with respect to the Bootstrap model captures the diversification effect by returning a much larger variability for the smallest insurer while shrinking it for the largest one. A less prominent effect is produced on skewness. Differences are partly due to the different assumptions implicitly included in the F-L and CL deterministic methods, both of which are applied here without any "professional judgement" (which instead is always present in real valuations). This may lead to different averages on both discounted and undiscounted bases. For simplicity's sake, results based on different probabilistic assumptions (e.g. LogNormal, ODP, Normal and Negative Binomial) are not reported in this paper. In general, for Bootstrap we have obtained a skewness ranging from 0.05 to 0.08 for SIFA and from 0.078 to 0.085 for AMASES. On the other hand, CRM returns a significantly different skewness for the two insurers, with a systematically lower value for the larger company 
because of its larger number of paid claims.

Table 1. Comparison of CV and skewness of the claim reserve distribution derived from both CRM and ODP Bootstrap

\begin{tabular}{lcccc}
\hline & \multicolumn{3}{c}{ CV } & \multicolumn{2}{c}{ Skewness } \\
\hline & CRM (F-L) & ODP Bootstrap (C-L) & CRM (F-L) & ODP Bootstrap (C-L) \\
\hline SIFA & 0.087 & 0.046 & 0.15 & 0.08 \\
AMASES & 0.033 & 0.037 & 0.06 & 0.08 \\
\hline
\end{tabular}

Note: 50,000 simulations - discounted values.

We have also evaluated how our model behaves over a one-year time horizon, as requested under the Solvency II framework, by comparing it with the most widely used methodology, One-Year Bootstrap. In this regard, Figure 2 reports the quantiles of Claims Development Result (CDR) distributions derived by both methods ${ }^{\mathrm{x}} . \overline{C D R}$ is usually defined as $\widetilde{C D R}=R_{0}-v_{1} \tilde{R}_{1}^{-}$and measures how far the discounted insurer obligation at the end of the following year is from the initial reserve. In other words, it measures profit $(C D R>0)$ or losses $(C D R<0)$ after the reserving process. As partially shown also by the characteristics of the Reserve distributions (see Table 2), CRM and Bootstrap provide similar distributions when the large insurer is considered. However, of note is the heavier left tail when Bootstrap is applied, which leads to a greater capital requirement. ODP Bootstrap provides instead a significant underestimation of extreme losses for SIFA Insurer.
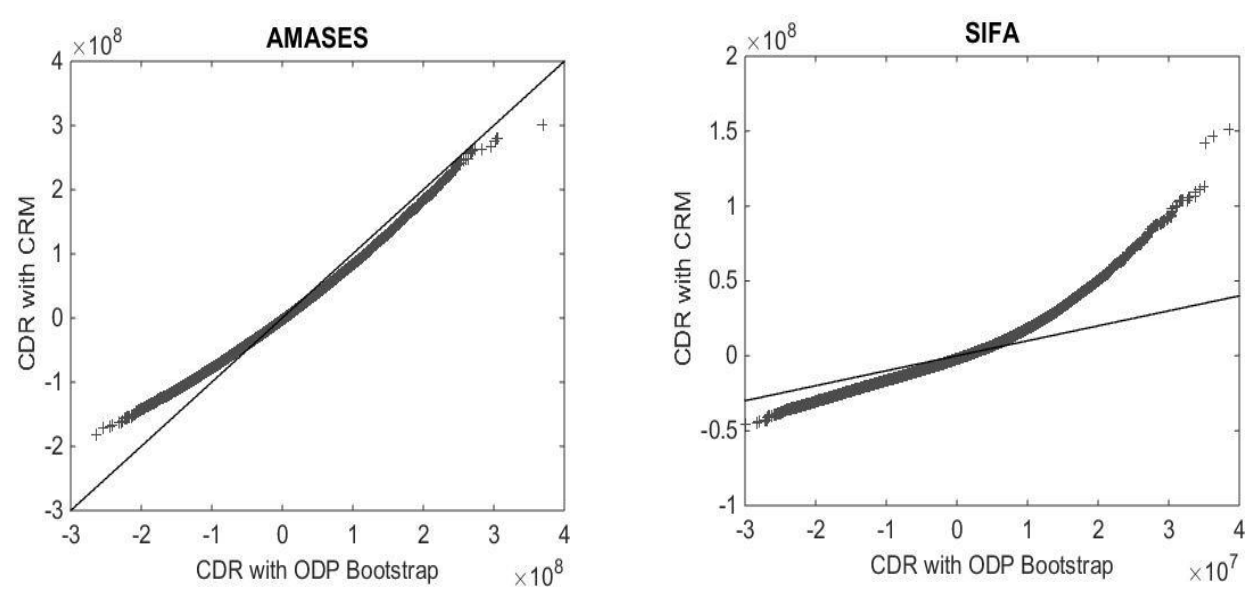

Figure 2. qqplot of CDR distributions derived from CRM and ODP Bootstrap

Table 2 shows the ratios between SCR for reserve risk and the Best Estimate of Loss Reserve, obtained using ODP Bootstrap and CRM for both insurers based on three different confidence levels. The benchmark is the $99.5 \%$ confidence level adopted by the Solvency II Directive. The capital requirement is derived from the left tail of the CDR distribution in (6) (i.e. $S C R^{99.5 \%}=-\operatorname{VaR}_{0.5 \%}(\widetilde{C D R})$ ). As expected, the smaller insurer has a higher SCR ratio due to the more skewed claims reserve distribution. Furthermore, differences between models are more significant for SIFA Insurer and for higher confidence levels because of the skewness effect.

Table 2. SCR ratio obtained by CRM and ODP Bootstrap for both insurers with a One-Year time horizon

\begin{tabular}{lcccccc}
\hline & \multicolumn{5}{c}{$S C R_{0} / B E_{0}$} \\
\hline & CRM(F-L) & \multicolumn{4}{c}{ ODP Bootstrap (C-L) } \\
\hline SIFA & $99.00 \%$ & $99.50 \%$ & $99.97 \%$ & $99.00 \%$ & $99.50 \%$ & $99.97 \%$ \\
AMASES & $22.4 \%$ & $26.4 \%$ & $44.4 \%$ & $9.1 \%$ & $10.1 \%$ & $13.6 \%$ \\
\hline & $5.8 \%$ & $6.6 \%$ & $10.0 \%$ & $6.7 \%$ & $7.5 \%$ & $10.3 \%$ \\
\hline
\end{tabular}

Note: 50,000 simulations - discounted values.

Focusing only on the benchmark level of $99.5 \%$, Figure 3 shows the SCR ratio obtained by several approaches in order to verify if an internal model may permit the saving of capital compared to the standard formula. In particular, besides the internal models described above (ODP Bootstrap and CRM), capital has been quantified 
by the Delegated Acts (DA) standard formula (European Commission, 2015) through either the market-wide or one of the several specific undertaking approaches. A market-wide capital charge is determined as three times the volatility factor $\sigma$, provided for each $\operatorname{LoB}$ (equal to $9.5 \%$ for MTPL), and multiplied by the volume measure ${ }^{\mathrm{xi}}$.

As expected, both insurers have the same capital ratios obtained from the market-wide formula. Moreover, DA allows us to estimate the volatility factor through a credibility structure equal to a weighted average of the market-wide volatility factor and a specific estimate of $\sigma$ (known as the undertaking-specific approach - USP). For this specific estimate of reserve risk variability, the approach based on Wüthrich and Merz formula (Wüthrich and Merz, 2008) is applied here ${ }^{\text {xii }}$.

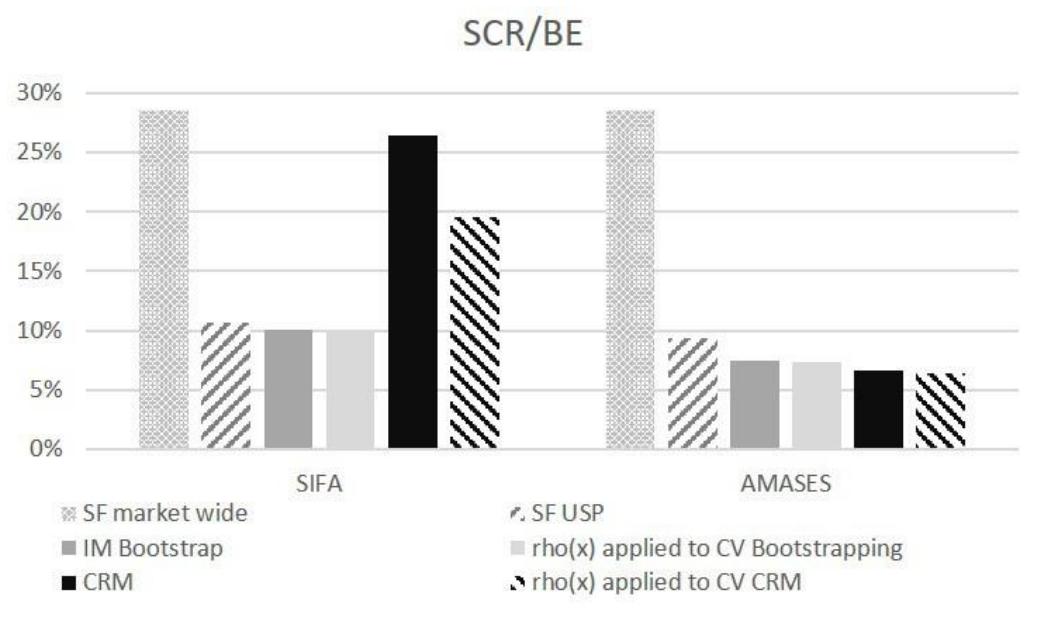

Figure 3. SCR ratio derived from different methodologies

It is noteworthy that the USP approach gives a significant saving of capital for both insurers with respect to the market-wide formula and that this approach is not so far from the results obtained by the ODP Bootstrap method. The CRM model leads instead to a SCR ratio similar to a market-wide standard formula for SIFA and to a capital amount for AMASES that is lower than that resulting from USP and bootstrapping.

Figure 3 also aims to check the impact of the Lognormal distribution assumption regarding claims reserve in the standard formula. In this regard, we quantify the capital requirement by applying the $\rho(x)$ function to the coefficient of variation obtained respectively by the Bootstrap and CRM model. As in premium risk valuation, it is confirmed that the LogNormal (with only two parameters) the underlying random variable has a significant positive skewness.

The CRM model has also been applied to other LoBs by exploring both the total run-off and the one-year approach. In particular, two relevant lines of business, General Third-Party Liabilities (GTPL) and Motor Own Damages (MOD), have been analyzed. For simplicity's sake, we report only the main results in terms of quantiles and capital requirement ratio (see Figure 4 and 5). 

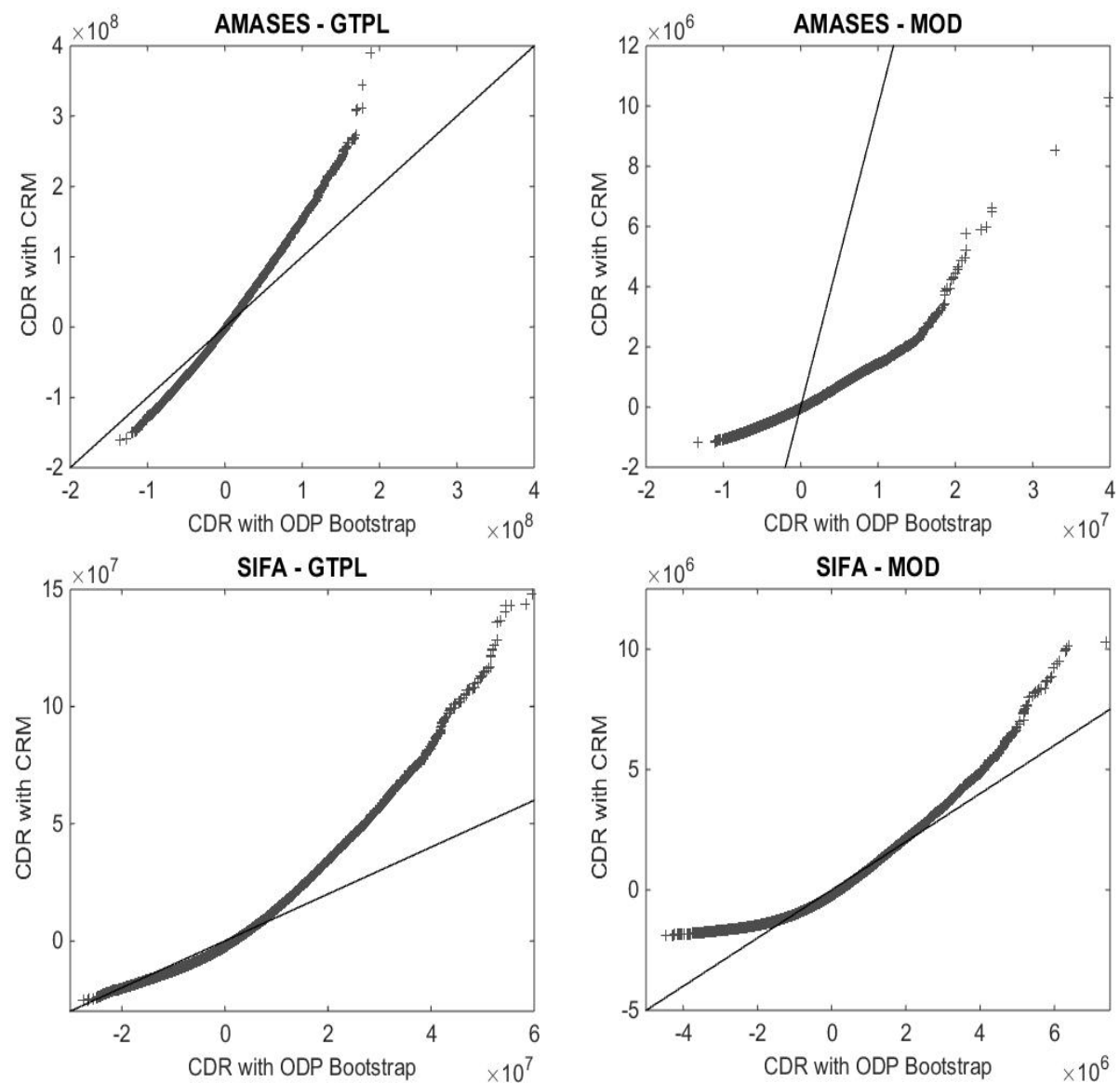

Figure 4. qqplot of CDR distributions derived from CRM and ODP Bootstrap for GTPL and MOD
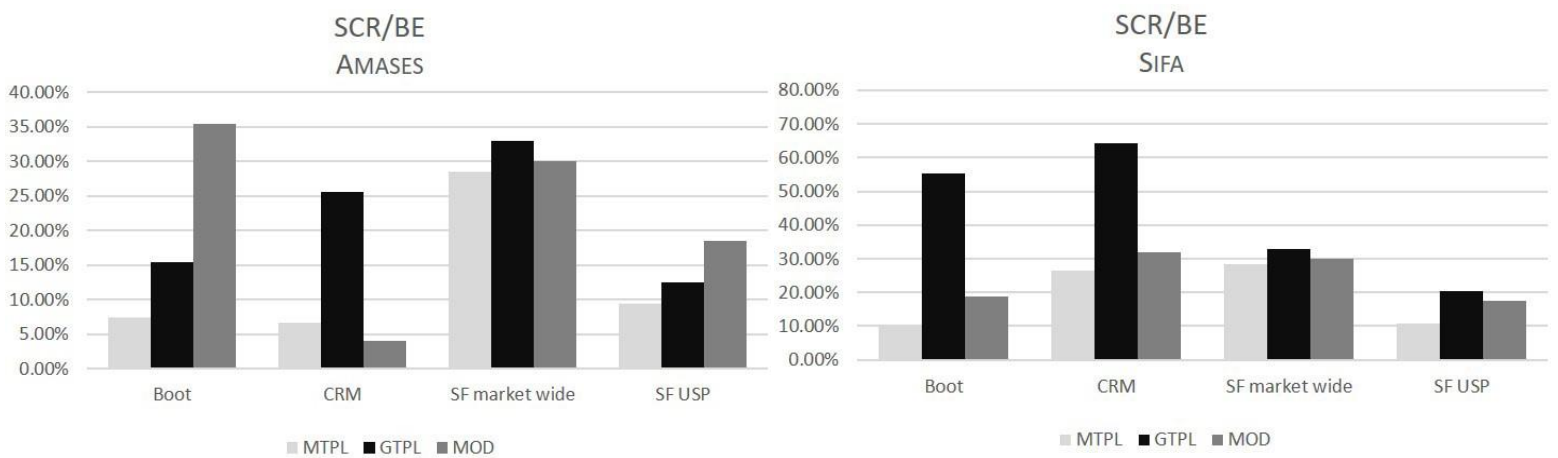

Figure 5. SCR ratios for MTPL, GTPL and MOD (partial internal models vs DA standard formula)

First of all, the CRM model shows consistent results when compared to classical methodologies, as it represents a viable alternative for deriving the distribution of claims reserve. In particular, as expected all approaches (simulations and closed formulae) confirm the greater dispersion for the GTPL line. In fact, GTPL provides a higher SCR ratio than MTPL because of more relevant CV and skewness. Moreover, it is noteworthy that the standard formula (GTPL market-wide volatility factor is equal to $11 \%$ ) leads to a lower capital requirement than does the internal model (either ODP Bootstrap or CRM) for SIFA. Only the USP approach guarantees a saving of capital because of the very low CV. The larger company instead has lower requirements both for IM and USP along with greater savings when using methodologies based on the Chain-Ladder assumptions. As regards the MOD, models based on Chain-Ladder assumptions (such as ODP Bootstrap and the Wüthrich-Merz Formula) provide higher SCR ratios for AMASES. On the contrary, CRM reflects the dimensional effect, returning a 
negligible capital for AMASES and a ratio for SIFA higher than that provided by the MW approach. The standard formula, based on a 10\% volatility factor, gives lower requirements (see CRM for SIFA and Bootstrap for AMASES). This LoB clearly shows how the shape of the distribution could be very different by varying the models. In this case, the assumptions of the models are heavily affected by the data used. For example, considering the case of AMASES, the volatility of development factors, used by Bootstrap, leads to a more volatile distribution, which is not caught by the CRM model. Having at our disposal the marginal distributions, the aggregate capital requirement can also be derived by using either a correlation approach, as provided by QIS and the DA Standard Formula, or copula functions, in order to take into account tail dependencies, which are usually not negligible in this context (for details see Savelli and Clemente (2011)). We could finally obtain the aggregate SCR for both companies. Table 3 summarizes the loss reserve distribution showing a prominent weight of MTPL and a greater weight of MOD for the small Insurer. The Solvency II Standard Formula proposes an aggregation of SCR based on the given correlation matrix (see Table 4 from European Commission (2015) p.255 Annex IV).

Table 3. Share of Total Best Estimate

\begin{tabular}{|c|c|c|c|c|}
\hline & MTPL & GTPL & MOD & Total \\
\hline SIFA & $69.7 \%$ & $24.1 \%$ & $6.2 \%$ & $100.0 \%$ \\
\hline AMASES & $76.6 \%$ & $22.3 \%$ & $1.1 \%$ & $100.0 \%$ \\
\hline \multicolumn{5}{|c|}{ Table 4. Correlation matrix } \\
\hline & MTPL & & GTPL & MOD \\
\hline MTPL & 1 & & 0.5 & 0.5 \\
\hline GTPL & 0.5 & & 1 & 0.25 \\
\hline MOD & 0.5 & & 0.25 & 1 \\
\hline
\end{tabular}

We compare this solution with copula functions calibrated to assure comparable correlation coefficients. Not having at disposal the time series of CDR of the three LoBs, we used the correlation matrix reported in Table 4. In this case, we are not focused on the calibration of copula functions, but even for practical purposes we aggregate CDR distributions providing a consistent comparison between internal model and standard formula. In particular, two classical elliptical copulas (Gaussian and t-Student) and two classical Archimedean copulas (Clayton and Mirror Gumbel ${ }^{\mathrm{xiv}}$ ) are applied. We test the latter class to consider the tail dependency contribution to the aggregation process. Furthermore, it is well-known that this class of copulas has one generating function and usually one parameter that characterizes the dependence structure of the joint distribution function; however, the use of only one parameter could be extremely restrictive in a higher-dimensional case. A two-level hierarchy of Archimedean copulas is applied here to solve this issue. In this specific case, first the aggregated distribution between MOD and GTPL lines has been obtained and then the result has been aggregated with the distribution of MTPL. Figure 6 compares the aggregated distribution of CDR derived for each model and company by using Gaussian and Clayton Copula. As expected, there is a significant fat left tail when the Clayton Copula is used. Furthermore, of note is the lower volatility of the aggregate distribution of SIFA when ODP-Bootstrap is applied. 

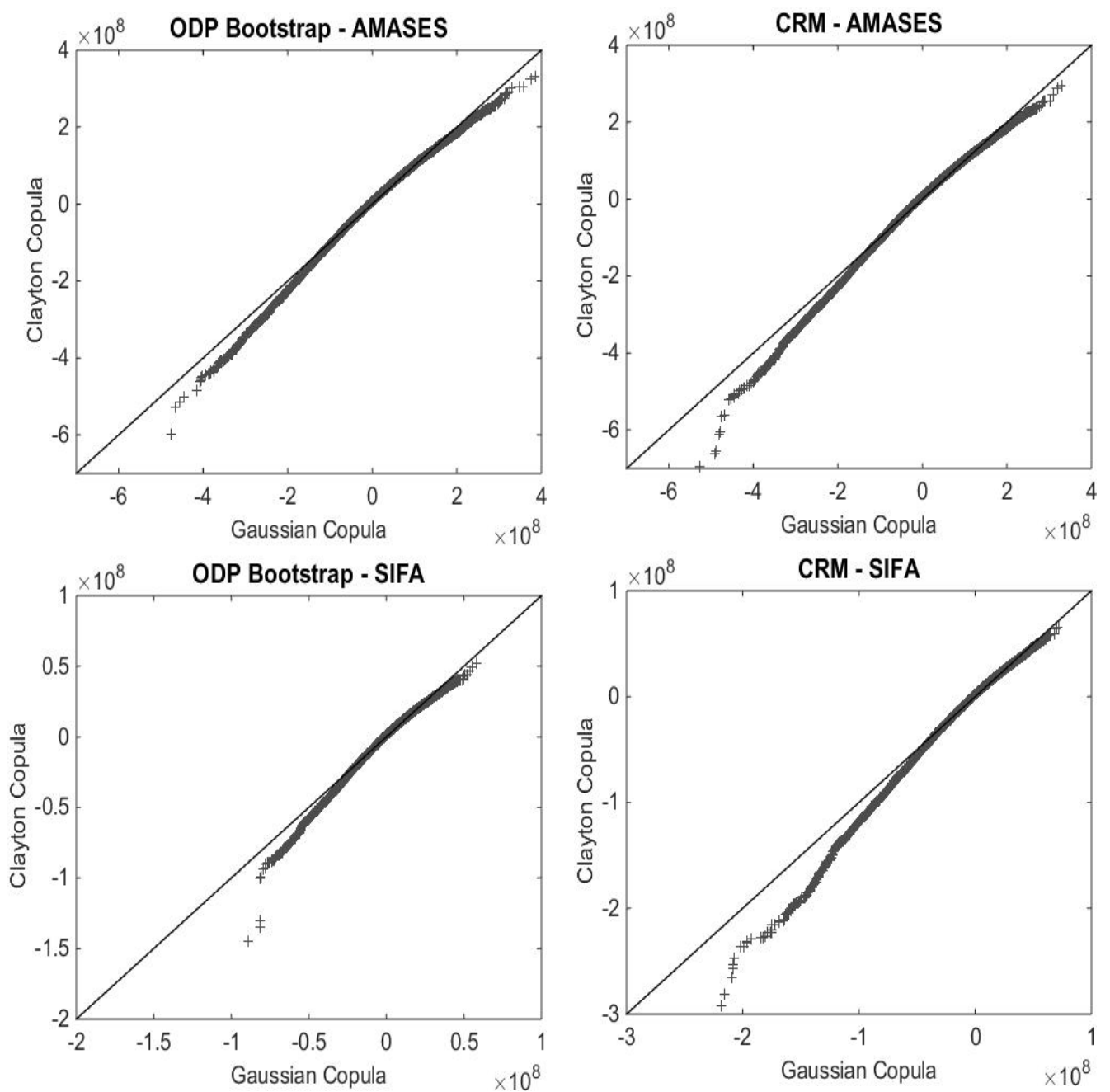

Figure 6. qqplot of Aggregate CDR distributions derived from Gaussian and Clayton Copulas

In terms of the capital requirement, Figure 7 shows how both Internal Models (ODP and CRM) lead to similar capital requirements for AMASES and a significant savings in the capital requirement with respect to the MW approach of the Standard Formula. On the other hand, the Chain Ladder-based approaches (Standard Formula USP and ODP-Bootstrap) tend to underestimate the capital requirements when SIFA is considered.

SIFA

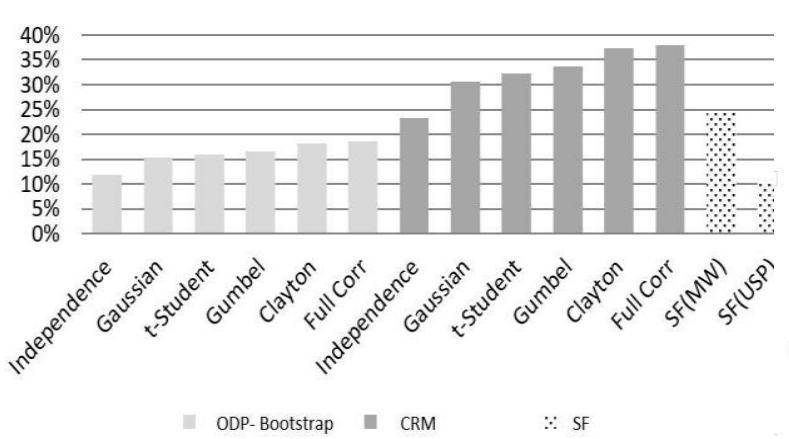

AMASES

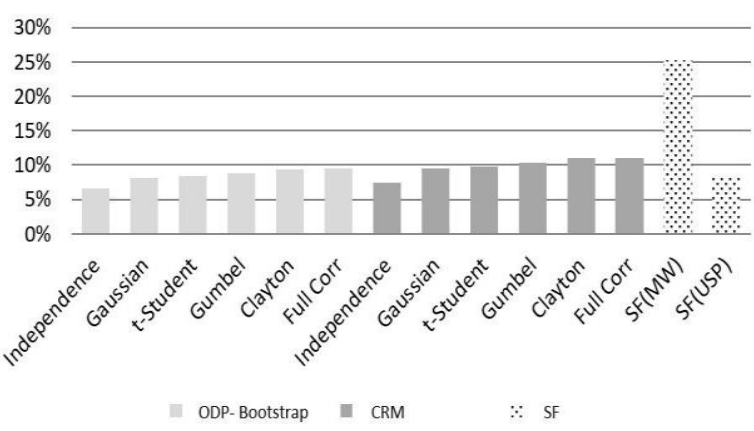

Figure 7. SCR ratios derived from different methodologies 


\section{Conclusions}

The CRM model applied to each cell of the run-off triangles may represent a viable alternative to the stochastic models for reservation available in the literature. Parameter estimates may be obtained using claims DataBase and an average cost method that provides separate estimates for the number of future payments and average claim costs. Our approach allows us to customize the stochastic process of each time event with its proper distribution function by means of an appropriate fitting procedure. This extends the capability of the standard CRM by solving the not-rare cases where some years are affected by specific events or commercial strategies. The LoBs analyzed in the case study show that CRM has a different impact with respect to ODP Bootstrap. In general, CV and skewness of claims reserve distribution are more sensitive to insurer size when the CRM model is applied, as would be expected. Moreover, by aggregating LoBs using the copula methodology we have shown how CRM sheds light on the uncertainty of future costs, especially when small volume companies are analyzed. Some sensitivities show the relevance of having a reliable parameter calibration and the strict link between parameter uncertainty and the variability of the overall claim reserve. In particular, attention should be paid to the estimation of the standard deviation of the structure variable $\tilde{q}$ in order to properly capture the correlation inside the triangle. The coefficient of variation of the total run-off claims reserve distribution is significantly affected by that value. The One-Year approach represents a key approach to be fully investigated in order to perform evaluations consistent with Solvency II. If CRM is used for reserve risk, a strong consistency with the premium risk modelling might result when appropriate. Further research will regard methods to introduce stochastic inflation along with analyses to evaluate the effect of tail factors on CRM and on both the One-Year and Total Run-Off approaches.

\section{Acknowledgments}

We would like to thank the associate editor and two anonymous referees for their careful reading and constructive suggestions. Funding for this research was provided by Università Cattolica del Sacro Cuore.

\section{References}

Alai, D. H., Merz, M., \& Wüthrich, M. V. (2009). Mean square error of prediction in the Bornhuetter-Ferguson claims reserving method. Annals of Actuarial Science, 4(1), 7-31. https://doi.org/10.1017/S1748499500000580

Avanzi, B., Taylor, G., Vu, P. A., \& Wong, B. (2016). Stochastic loss reserving with dependence: A flexible multivariate Tweedie approach. Insurance, Mathematics and Economics, 71, 63-78. https://doi.org/10.1016/j.insmatheco.2016.08.006

Daykin, C. D., Pentikäinen, T., \& Pesonen, M. (1993). Practical risk theory for actuaries. Chapman and Hall/CRC.

Diers, D. (2009). Stochastic re-reserving in multi-year internal models. Astin Colloquium.

England, P. (2002). Addendum to "Analytic and bootstrap estimates of prediction errors in claims reserving". Insurance, Mathematics and Economics, 31(3), 461-466. https://doi.org/10.1016/S0167-6687(02)00161-0

England, P. D., \& Verrall, R. J. (2002). Stochastic claims reserving in general insurance. British Actuarial Journal, 8(3), 443-518. https://doi.org/10.1017/S1357321700003809

England, P. D., \& Verrall, R. J. (2006). Predictive distributions of outstanding liabilities in general insurance. Annals of Actuarial Science, 1(2), 221-270. https://doi.org/10.1017/S1748499500000142

England, P., \& Verrall, R. (1999). Analytic and bootstrap estimates of prediction errors in claims reserving. Insurance, Mathematics and Economics, 25(3), 281-293. https://doi.org/10.1016/S0167-6687(99)00016-5

England, P., Verrall, R., \& Wüthrich, M. (2012). Bayesian Overdispersed Poisson Model and the Bornhuetter-Ferguson Claims Reserving Method. Annals of Actuarial Science, 6(2), 258-283. https://doi.org/10.1017/S1748499512000012

European Commission (2009). European Parliament legislative resolution of 22 April 2009 on the amended proposal for a directive of the European Parliament and of the Council on the taking-up and pursuit of the business of Insurance and Reinsurance (recast), Directive 2009/138/EC. Technical report, European Commission.

European Commission (2010). Quantitative Impact Study 5 - Technical Specifications. Technical report, European Commission.

European Commission (2013). Directive of the European Parliament and of the Council amending Directive 
2009/138/EC (Solvency II) as regards the date for its transposition and the date of its application, and the date of repeal of certain Directives (Solvency I), Directive 2013/58/EU. Technical report, European Commission.

European Commission (2015). European Commission (2015) Commission Delegated Regulation (EU) 2015/35 supplementing Directive 2009/138/EC of the European Parliament and of the Council on the taking-up and pursuit of the business of Insurance and Reinsurance (Solvency II), 10 of October 2014. Technical report, Official Journal of the European Union.

Fisher, W. H., \& Lange, J. T. (1973). Loss reserve testing: a report year approach. In Proceedings of the Casualty Actuarial Society, 60, 189-207.

Friedland, J. (2010). Estimating unpaid claims using basic techniques. In Casualty Actuarial Society, volume 201.

International Actuarial Association (2004). A Global Framework for Insurer Solvency Assessment. Technical report, Research Report of the Insurer Solvency Assessment Working Party.

Klugman, S., Panjer, H. H., \& Willmot, G. E. (2008). Loss Models: from data to decisions, Wiley. https://doi.org/10.1002/9780470391341

Mack, T. (1993). Distribution-free calculation of the standard error of chain ladder reserve estimates. ASTIN Bulletin, 23(2), 213-225. https://doi.org/10.2143/AST.23.2.2005092

Martinez-Miranda, M. D., Nielsen, J. P., Verrall, R., \& Wüthrich, M. V. (2015). Double chain ladder, claims development inflation and zero-claims. Scandinavian Actuarial Journal, 2015(5), 383-405. https://doi.org/10.1080/03461238.2013.823459

Meyers, G. (2008). Stochastic Loss Reserving with the Collective Risk Model. Technical report, Casualty Actuarial Society.

Meyers, G., Klinker, F., \& Lalonde, D. (2003). The aggregation and correlation of Insurance risk. Technical report, Casualty Actuarial Society.

Ohlsson, E., \& Lauzeningks, J. (2008). The One-Year Non-Life Insurance Risk. Astin Colloquium.

Ottaviani, R. (1983). Metodologie ed analisi statistiche nel settore delle assicurazioni. In Proceedings of Italian Society of Statistics (SIS).

Peters, G. W., Shevchenko, P. V., \& Wüthrich, M. V. (2009). Model uncertainty in claims reserving within Tweedie compound Poisson models. ASTIN Bulletin: The Journal of the IAA, 39(1), 1-33. https://doi.org/10.2143/AST.39.1.2038054

Pinheiro, P. J., Andrade e Silva, J. M., \& de Lourdes Centeno, M. (2003). Bootstrap methodology in claim reserving. Journal of Risk and Insurance, 70(4), 701-714. https://doi.org/10.1046/j.0022-4367.2003.00071.x

Renshaw, A. E., \& Verrall, R. J. (1998). A stochastic model underlying the chain-ladder technique. British Actuarial Journal, 4(4), 903-923. https://doi.org/10.1017/S1357321700000222

Saluz, A., Gisler, A., \& Wüthrich, M. (2011). Development Pattern and Prediction Error for the Stochastic Bornhuetter-Ferguson Claims Reserving Method. Astin Bullettin, 41, 2.

Savelli, N., \& Clemente, G. P. (2010). A collective risk model for claims reserve distribution. In Proceedings of Risk-Theory Workshop, 59-87.

Savelli, N., \& Clemente, G. P. (2011). Hierarchical Structures in the aggregation of Premium Risk for Insurance Underwriting. Scandinavian Actuarial Journal, 3, 193-213. https://doi.org/10.1080/03461231003703672

Tee, L., Kärik, M., \& Viin, R. (2017). On Comparison of Stochastic Reserving Methods with Bootstrapping. Risks, 5(2), 1-21. https://doi.org/10.3390/risks5010002

Venter, G. (2002). Tails of copulas. Technical report, Casualty Actuarial Society.

Wüthrich, M. V. (2003). Claims reserving using Tweedie's compound Poisson model. ASTIN Bulletin, 33(2), 331-346. https://doi.org/10.1017/S0515036100013490

Wüthrich, M., \& Merz, M. (2008). Modelling the claims development result for Solvency purposes. In Casualty Actuarial Society E-Forum, 542-568. 


\section{Notes}

${ }^{\mathrm{i}}$ Bootstrapping usually gives a best estimate not exactly coincident with the "true mean" (obtained by the deterministic chain-ladder), since the mean of the adjusted Pearson residuals may not equal zero.

ii See England and Verrall (2006) for a procedure related to Mack's Bootstrapping model and for a version of ODP with non-constant scale parameters. See Pinheiro et al. (2003) for a version of Bootstrap based on Pearson residuals standardized with a factor depending on the diagonal of the "hat" matrix.

iii Random variables will be represented by a tilde over upper/lower cases. This is compliant with notation used in most actuarial studies.

iv To describe costs, Lognormal or Gamma distributions are generally used (see e.g. Klugman et al. (2008)). Often the best fit is obtained by applying a Gamma distribution to the claim costs. If the Lognormal is chosen, as its m.g.f. is not defined, results can be obtained only by approximation or simulation.

$\checkmark$ As regard the methodology of average cost assessment, the F-L method implicitly assumes that settlement of partial claims payment is consistent over time.

${ }^{v i}$ According to Article 76 of the Solvency II Directive European Commission (2009), the claims reserve must be equal to the current amount that insurance and reinsurance undertakings would have to pay if they were to transfer their insurance and reinsurance obligations immediately to another insurance or reinsurance undertaking. This definition leads to a claims reserve evaluated as the sum of the best estimate and risk margin. As prescribed by Solvency II (see European Commission (2015)), risk margin is not considered in (6) to avoid problems of circularity.

vii The effect of direct reimbursement (CARD), introduced in 2007 in the Italian Insurance Market, is not considered here.

viii The main stochastic approaches (as ODP Bootstrap, Merz and Wüthrich formula, etc.) are usually applied to triangles without a tail.

${ }^{\text {ix }}$ It may be shown that the choice of the claim-size distribution has a negligible impact on the variability of the reserve distribution but a large effect on skewness (see in this regard Savelli and Clemente (2010), Section 5). However, several analyses on empirical datasets show that the Gamma distribution is a satisfactory choice.

${ }^{x}$ Be aware that axis scale might be different for a matter of illustration.

${ }^{x i}$ Since QIS5 (European Commission, 2010), the capital requirement $(N L)$ for reserve risk was obtained jointly with premium risk as $N L=\rho(\sigma) V$, where $\rho(\sigma)$ is a function that measures the difference between the $99.5 \%$ quantile and the mean of a LogNormal distribution with a standard deviation equal to $\sigma$. Furthermore, $\mathrm{V}$ is the volume measure (equal to Best Estimate of Loss Reserve for reserve risk). Delegated Acts (European Commission, 2015) have replaced this formula by assuming $N L=3 \sigma \mathrm{V}$.

xii The approach has been proposed in QIS5 and also confirmed by European Commission (2015).

xiii For a LogNormal distribution, it could be proven that there is a strict relationship between the variability coefficient and skewness $\gamma(\tilde{X})=C V(\tilde{X})\left(3+C V^{2}(\tilde{X})\right)$.

${ }^{x i v}$ Mirror Gumbel is a copula with the same Kendall Tau and swapped tail concentration functions with respect to the original Gumbel copula, resulting in a heavy concentration of probability on left tail (see Venter (2002) and Savelli and Clemente (2011) for further details on flipping a copula).

\section{Copyrights}

Copyright for this article is retained by the author(s), with first publication rights granted to the journal.

This is an open-access article distributed under the terms and conditions of the Creative Commons Attribution license (http://creativecommons.org/licenses/by/4.0/). 